\title{
Research Paper: Comparison the Quality of Life Among Adults With and Without Stuttering: An Emphasis on the Severity of Stuttering
}

Seyed Sadegh Rahimi ${ }^{1}$, Morteza Farazi ${ }^{1}$, Akbar Darouie ${ }^{1}$, Enayatollah Bakhshi², Salman Abdi ${ }^{3}$, Vahid Valinejad ${ }^{1}$, Mohyeddin Teimori ${ }^{3}$

1. Department of Speech Therapy, University of Social Welfare and Rehabilitation Sciences, Tehran, Iran.

2. Department of Biostatistics, University of Social Welfare and Rehabilitation Sciences, Tehran, Iran.

3. Department of Speech Therapy, School of Rehabilitation, Tehran University of Medical Sciences, Tehran, Iran.

Received: 4 Apr. 2016 Accepted: 29 Jul. 2016

Keywords:

Quality of life, Adult who stutter, OASESA test, Stuttering severity
Citation: Rahimi SS, Farazi M, Darouie A, Bakhshi E, Abdi S, Valinejad V, et al. [Comparison the Quality of Life Among Adults With and Without Stuttering: An Emphasis on the Severity of Stuttering (Persian)]. Journal of Rehabilitation. 2017; 17(4):300-307. http://dx.doi.org/ 10.21859/jrehab-1704300

doi $)^{\text {: }}$ http://dx.doi.org/ 10.21859/jrehab-1704300 


\title{
مقايسه كيفيت زندگًى بزرتىسالان دجار لكنت با افراد بدون لكنت با تأكيد بر شدث لكنت
}

\author{
سيدصادق رحيمى'، "مرتضى فرازى'، اكبر دارويى'، عنايتالله بخشى'، سلمان عبدى"، وحيد ولىنزاد'، محى الدين تيمورى"
}

ا- كروه كفتاردرمانى، دانشكاه علوم بهزيستى و توانبخشى، تهران، ايران.

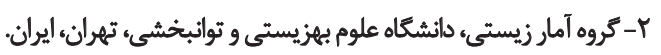

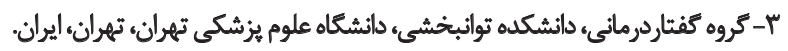

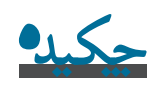

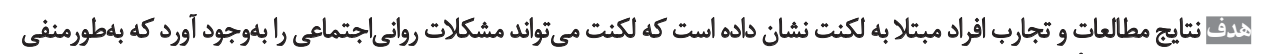

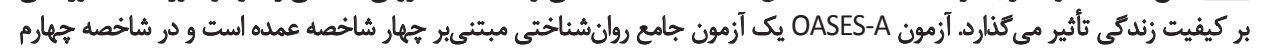

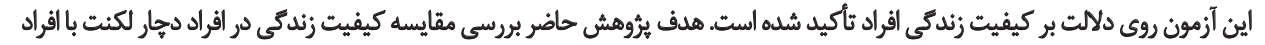

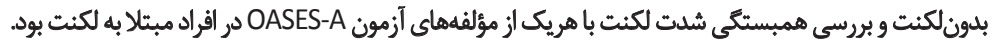

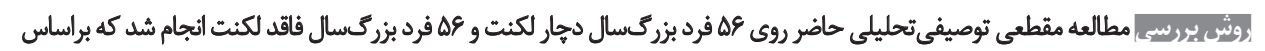

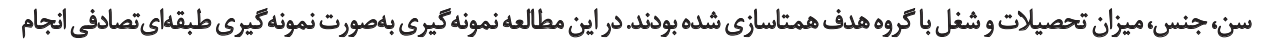

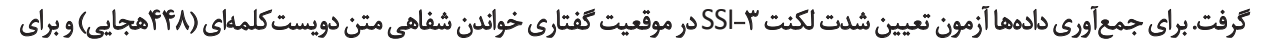

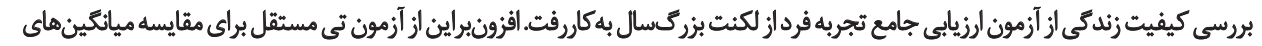

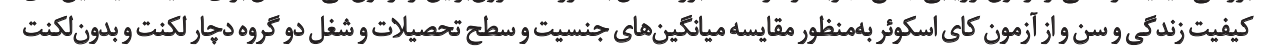

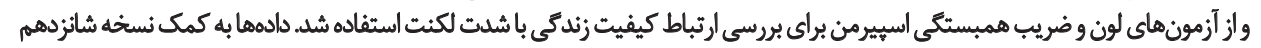

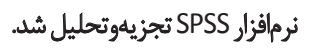

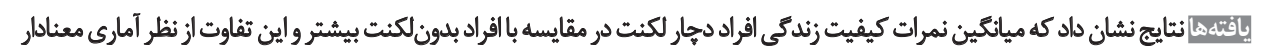

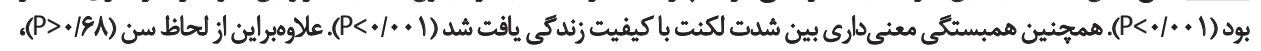

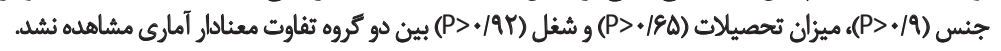

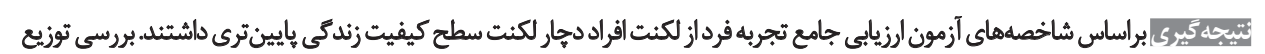

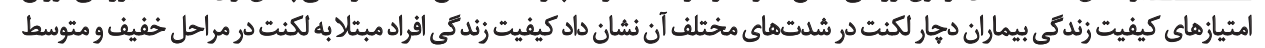

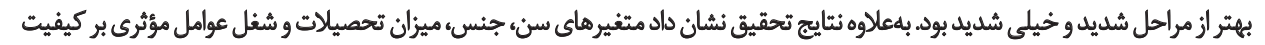

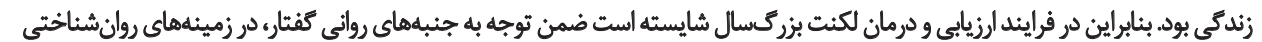

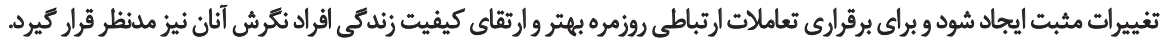

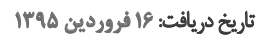

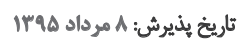

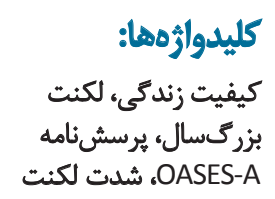

كليدوارهها:

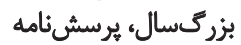
OASES-A

طريق كاهش آسيبهاى عملكردى و ساختارى بدن و محدوديت

مقدمه

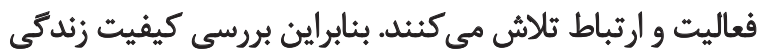

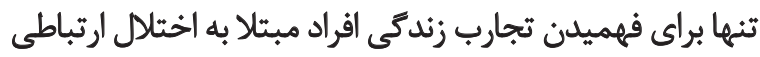

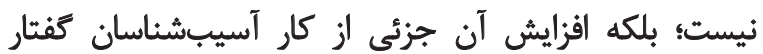

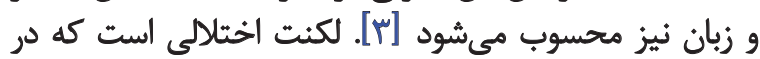

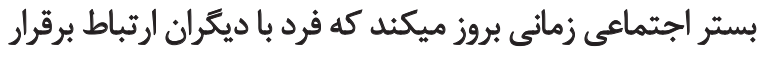

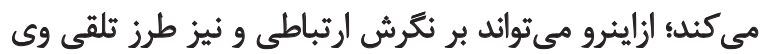

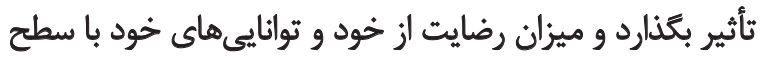

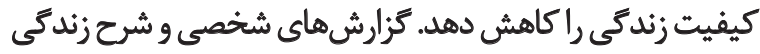

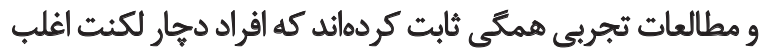

يكى از شيوههاى رايج و كامل برقرارى ارتباط انسانهاه از

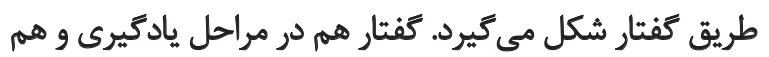

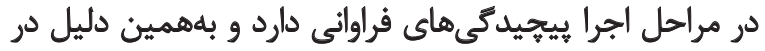

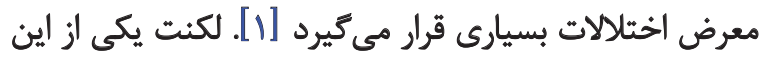

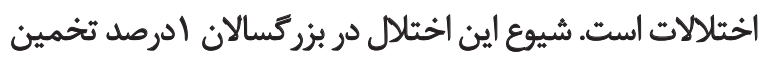

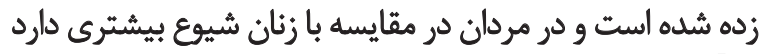

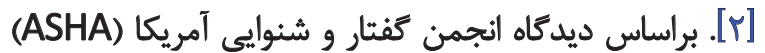

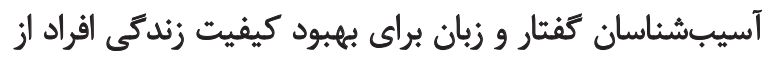




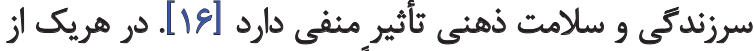

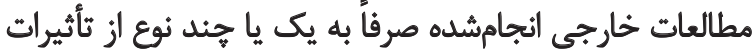

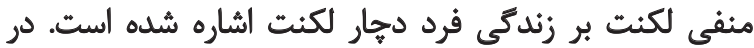

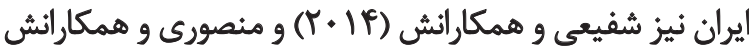

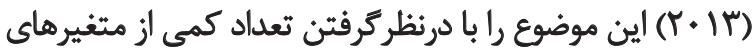

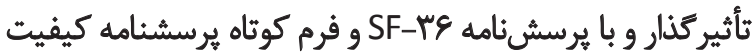

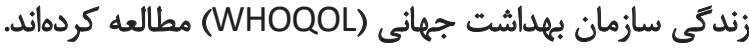

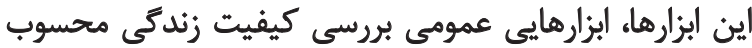

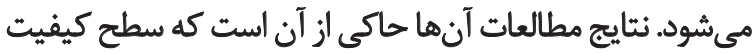

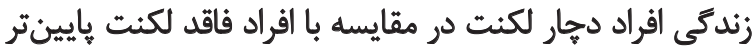

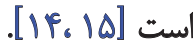

مطالعه حاضر با درك اهميت اين موضوع كيفيت زندكى إزي

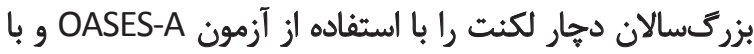

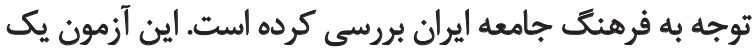

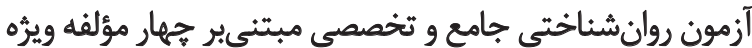

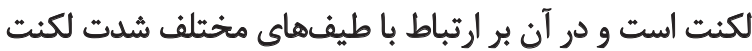

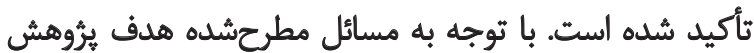

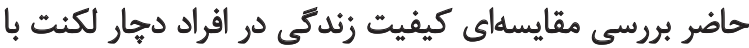

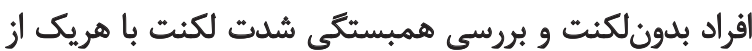

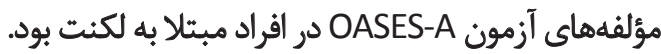

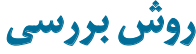

مطالعه حاضر، مطالعهاي توصيفىتحليلى بود كه بلهصورت

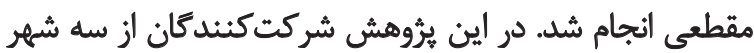

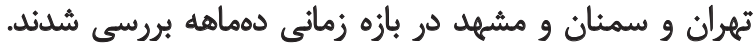

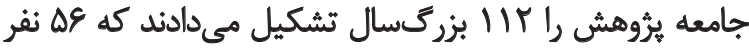

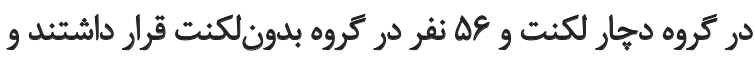

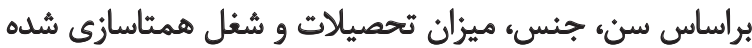

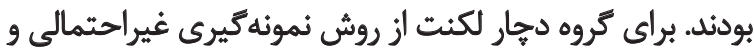

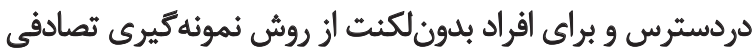

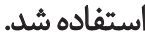

در مرحله اول بثروهشكر بامراجعه به كلينيكهاي تفتاردرمانى

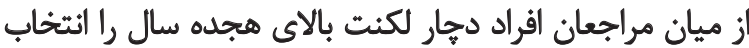

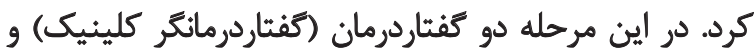

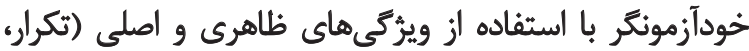

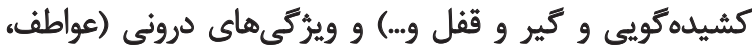

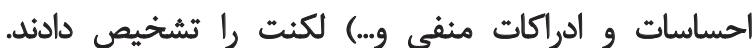

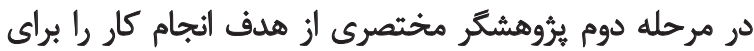

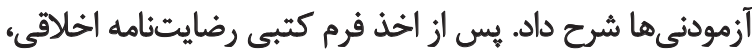

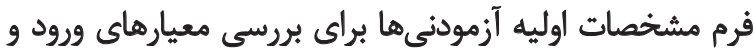

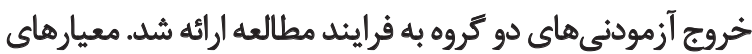

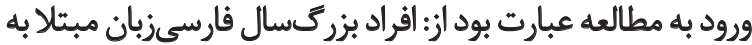
لكنت، سن بالاتر از هجده، حداقل بايه تحصيلى سوم راهنمائي،

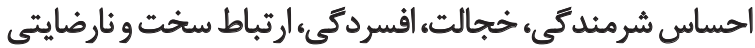

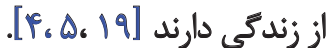

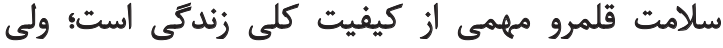

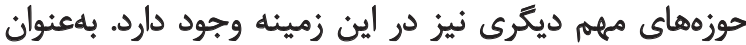

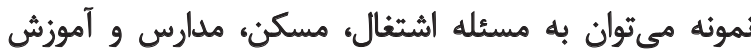

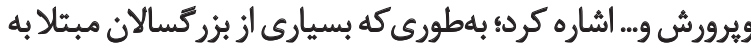

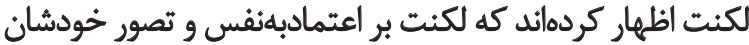

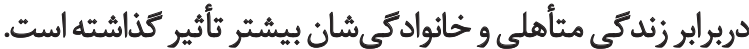

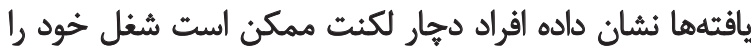

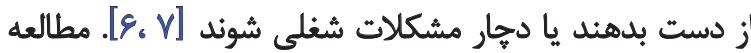

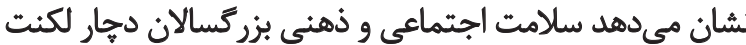

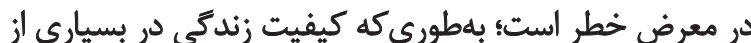

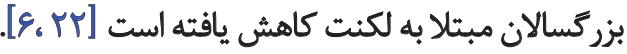

مطالب كفته شده نشان ميدهد كه بسيارى از افراد دجار

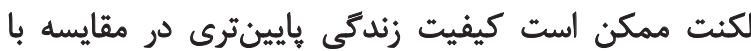

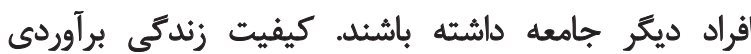

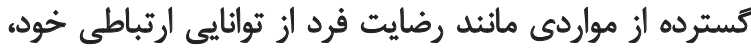

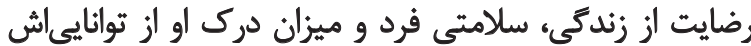

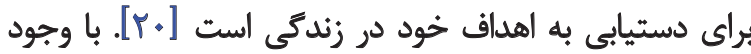

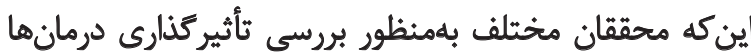

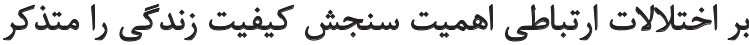

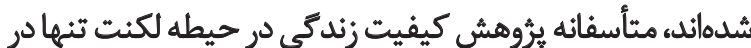

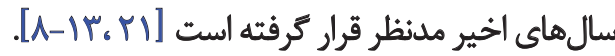

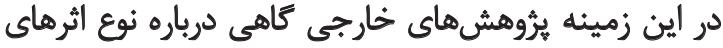

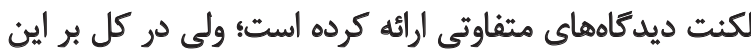

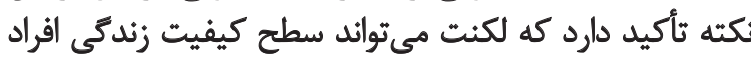

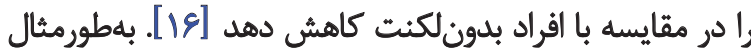

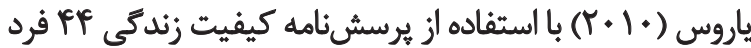

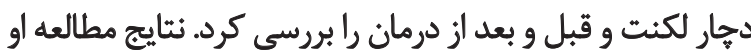

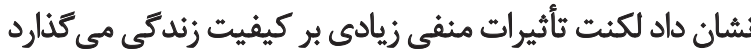

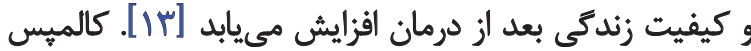

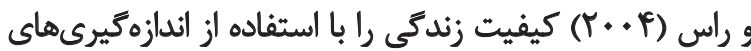

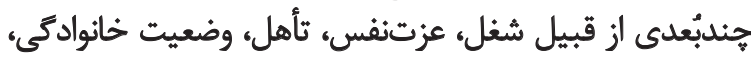

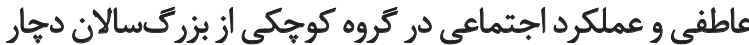

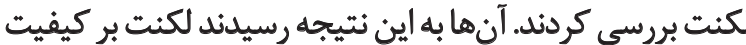

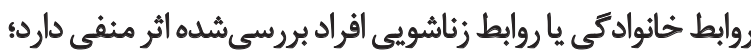

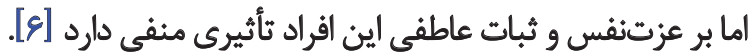

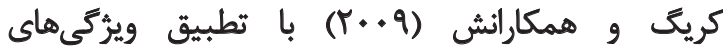

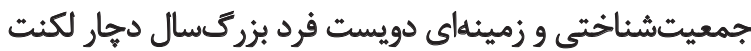

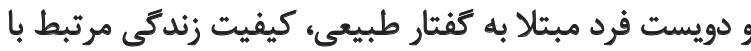

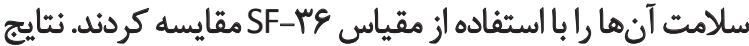

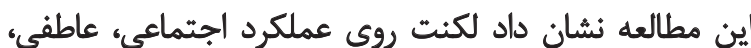


دادههاى هر آزمودنى يّ ازئ جمعآورى با استفاده از نسخه

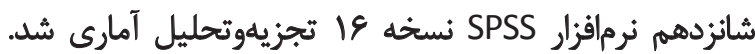

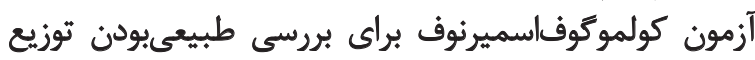

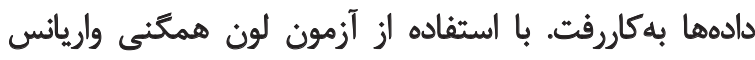

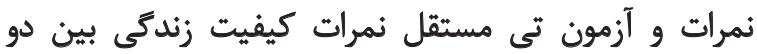

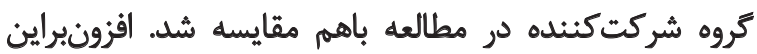

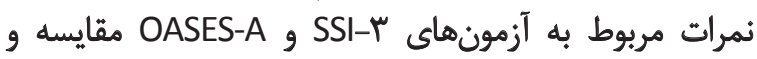

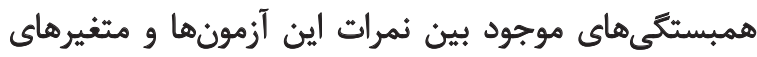

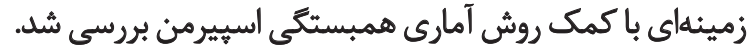

يافتهها

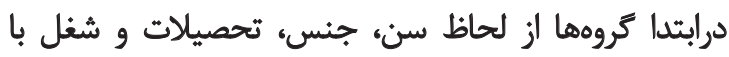

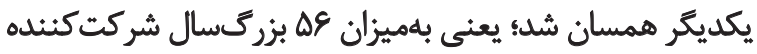

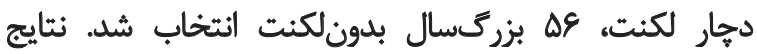

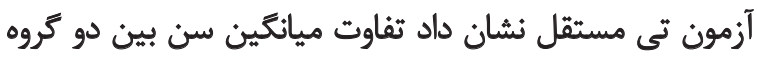

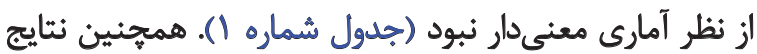

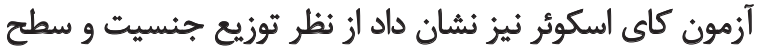

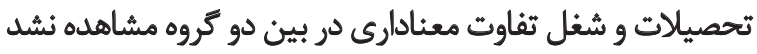

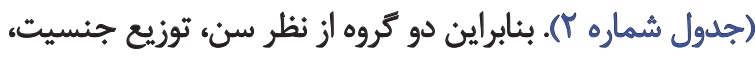
سطح تحصيلات و شغل مشابه بودند.

درادامه ميانكين نمرات كل OASES-A و جهار شاخصه آن

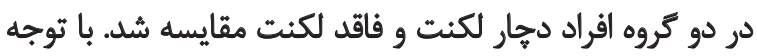

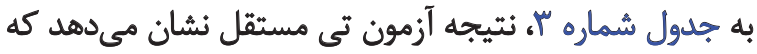

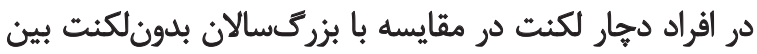

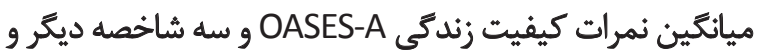

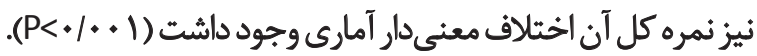

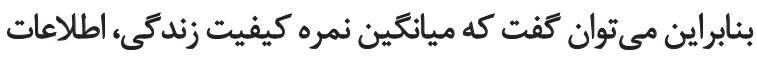

بيش از fادرصد هجاهاى الكنتشده (SS\%)'، نداشتن مشكل

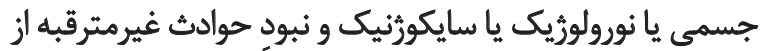

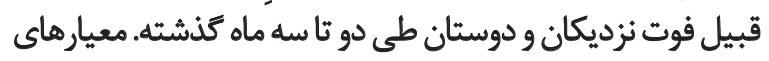

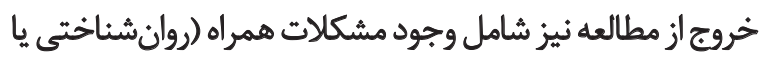

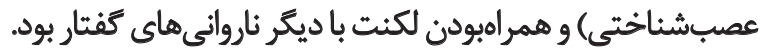

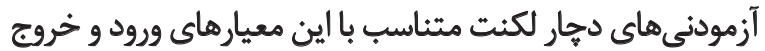
بر برسى شدند.

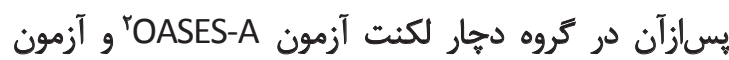

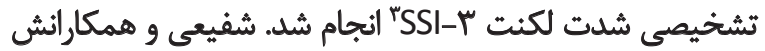

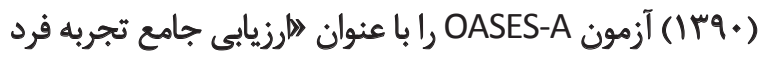

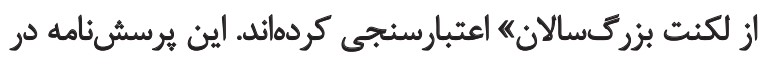

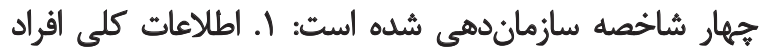

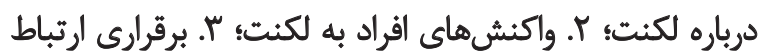

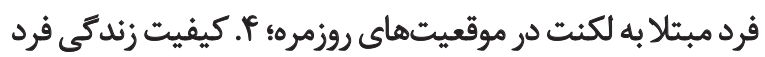

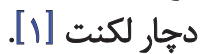

براى تعيين شدت لكنت از آزمون بـا SSI استفاده شد. براى

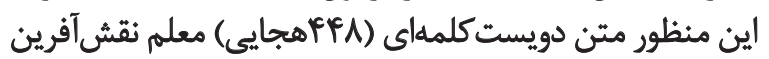

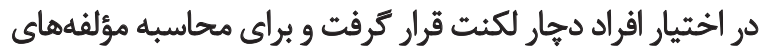

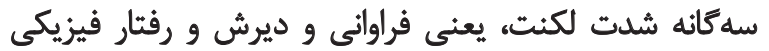

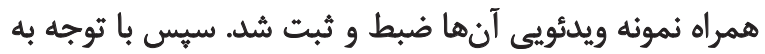

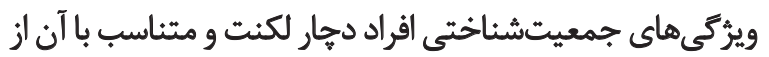

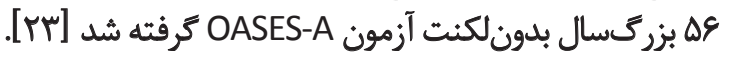

\section{Syllabled Stuttered}

2. Overall Assessment of the Speaker's Experience of StutteringAdult (A-SESAO)

3. Stuttering Severity Instrument-3 (SSI-3)

جدول ا. مقايسه ميانكين سن در دو كروه دجار لكنت و بدون لكنت.

\begin{tabular}{|ccccc}
\hline & & & \\
\hline
\end{tabular}

توانبخننى

جدول ץ. توزيع فراوانى دو كروه دجار لكنت و بدون الكنت برحسب جنسيث و سطح تحصيلات و شغل.

\begin{tabular}{|c|c|c|c|c|c|c|c|c|c|c|}
\hline \multicolumn{3}{|c|}{ شغل } & \multicolumn{3}{|c|}{ تحصيلات } & \multicolumn{2}{|c|}{ جنس } & \multirow[t]{2}{*}{ مثغير . } & \multirow{2}{*}{\multicolumn{2}{|c|}{ كروه }} \\
\hline أزاد & كارمند & محصل & بالاي دييلم & وييلم & زيردييلم & مونث & مذكر & & & \\
\hline Ir & 9 & $m$ & r. & M & is & $\wedge$ & is & & دهار لكثت & \\
\hline 10 & $\wedge$ & ra & ia & in & $\pi$ & $\wedge$ & is & & بدون لكنت & \\
\hline \multicolumn{3}{|c|}{$* \ldots$} & \multicolumn{3}{|c|}{.1 .01} & \multicolumn{2}{|c|}{$.1+\infty 1$} & & آماره آزمون كاي اسكوئر & \\
\hline \multicolumn{3}{|c|}{./QY } & \multicolumn{3}{|c|}{.180} & \multicolumn{2}{|c|}{.$/ 9$} & & مقدار احتمال & \\
\hline
\end{tabular}


جدول f. همبستكى بين نمره كل OASES-A و بخشهاى آن با شدت لكنت.

\begin{tabular}{|c|c|c|c|c|c|c|}
\hline مقدار احتمال & تمره كل & كيفيت زندكى & شاخصهها ارتباط & واكنشها & اطلاعات & مرات OASES-A \\
\hline$<+1 .+1$ & . /que & $+A r^{*}$ & $\cdot N \Delta^{\circ}$ & $\Rightarrow / M \Lambda^{\circ}$ & $\cdot / r^{\circ}$ & شدت لكنت \\
\hline
\end{tabular}

جدول "ا. توزيع نمرات آزمون OASES و شاخصههاى آن (كيفيت زئدىى) برحسب دو مروه دجار لكنت و بدونلكنت.

\begin{tabular}{|c|c|c|c|c|c|c|}
\hline \multirow[t]{2}{*}{$\cdot p$} & \multirow[t]{2}{*}{ آماره آزمون تى } & \multicolumn{2}{|c|}{ بدون ككثت } & \multicolumn{2}{|c|}{ دهار لكثت } & \multirow{2}{*}{ 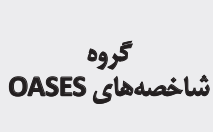 } \\
\hline & & اتحرافمعيار & ميانكين & اتحرافمعيار & ميانكين & \\
\hline$<+\%+1$ & $S / D A$ & var & $s \cdot 1$. & $1 . / \Delta r$ & $\Delta 1 / / 9$ & اطلاعاث كلى \\
\hline$<+1++1$ & R/RT & $1 / \pi e$ & $\mu / / q$. & $1 \Delta / \pi \varphi$ & $\Delta r / q r$ & واكثش به لكنت \\
\hline$<+1 \cdot+1$ & $\Delta / r T$ & $r 9 / 91$ & $|r / \Delta|$ & VAY & PNAS & برقرارى ارتباط \\
\hline$<\cdot \% 1$ & $15 / 99$ & re/N & $\mid V / . r$ & .199 & $\Delta r / a Y$ & كيفيت زندى \\
\hline$<+\%$ & $9 / 19$ & $P r / q$ & $11 / 29$ & $r M T$ & $\Delta / a r$ & نمره كل \\
\hline
\end{tabular}

براين اساس در ارزيابى نتايج درمان بايد علاوه بر ارزيابى تغييرات

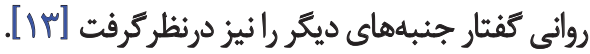

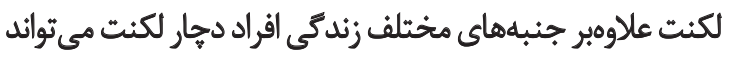

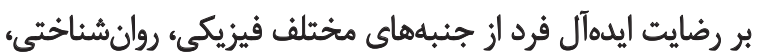

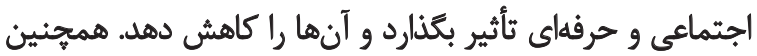

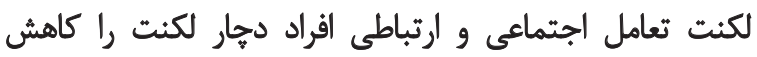

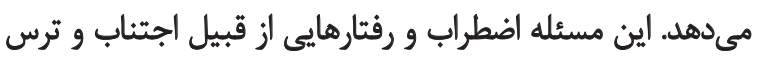

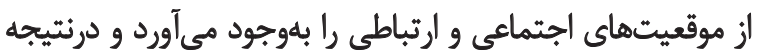

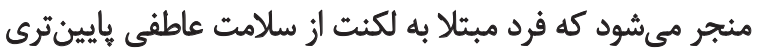
داشته باشد.

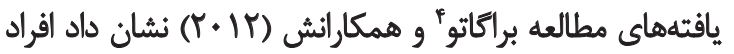

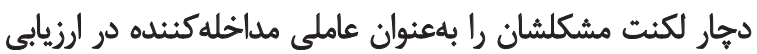

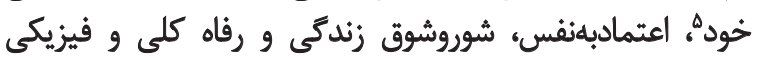

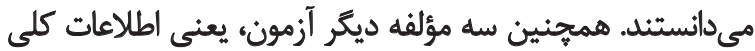

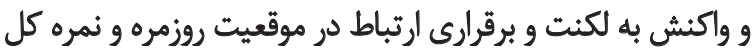

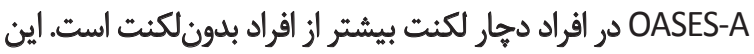

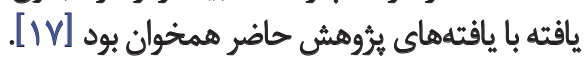

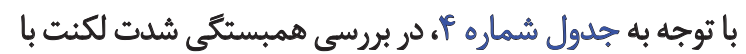

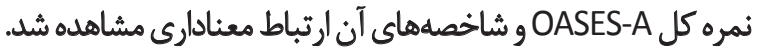

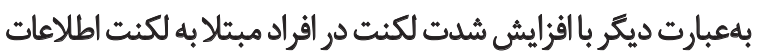

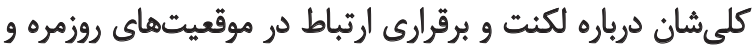

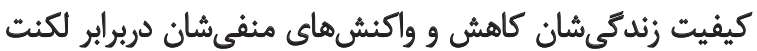

4. Bragatto

5. Self-esteem
كلى، واكنش به لكنت، برقرارى ارتباط در موقعيت روزمره و نمره

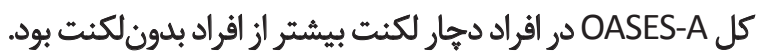
درنهايت ارتباط نمره كل OASES-A و جهار شاخصه آن با شدت

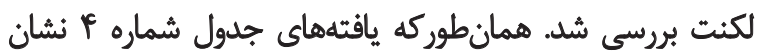

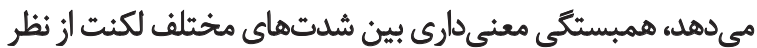

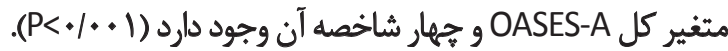

\section{بحث}

هدف مطالعه حاضر مقايسه نمرات كيفيت زندكى افراد دهار

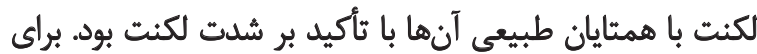

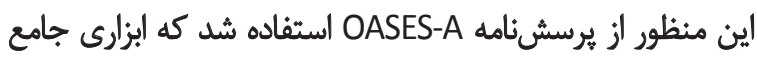

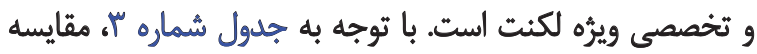

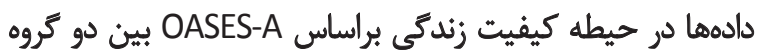

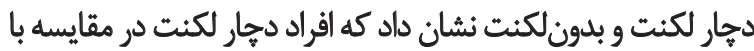

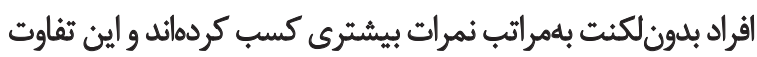

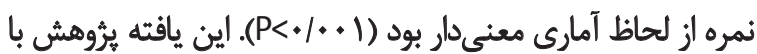

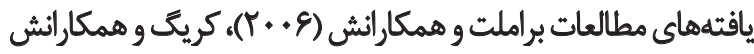

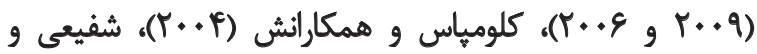

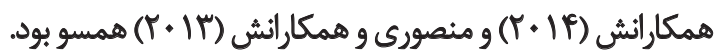

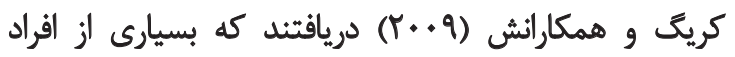

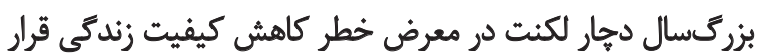

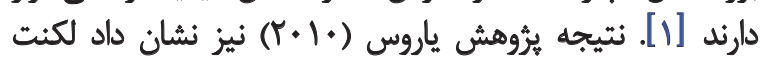

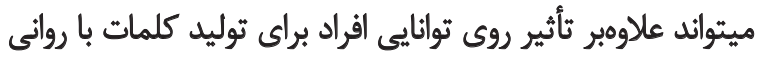

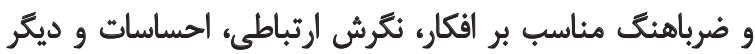

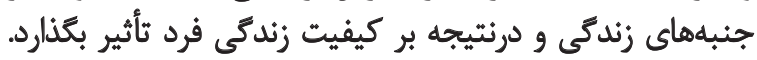




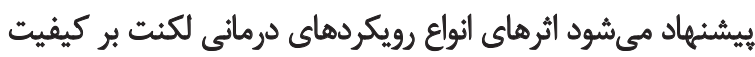

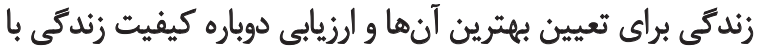

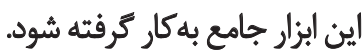

$$
\text { تشيكر و قعدرواني }
$$

اين مقاله حاصل بخشى از يايانانامه دوره كارشناسى ارشد

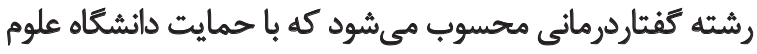

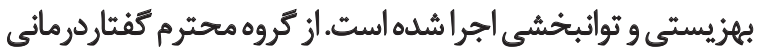

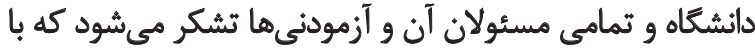
صبر و حوصله در اين مطالعه همكاري داشتئند.

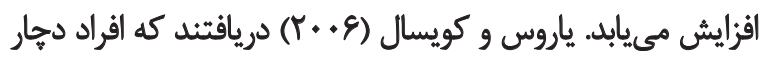

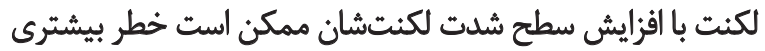

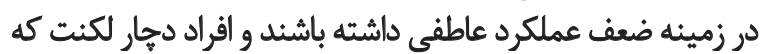

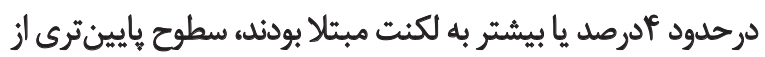

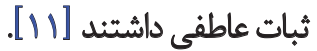

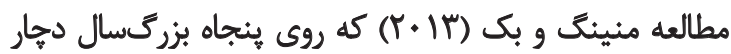

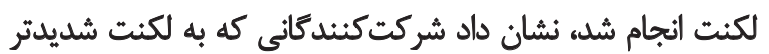

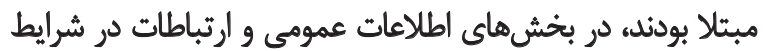

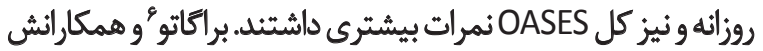

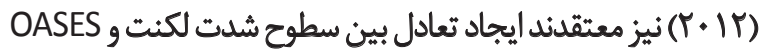

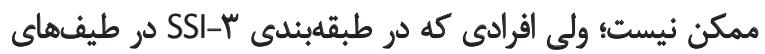

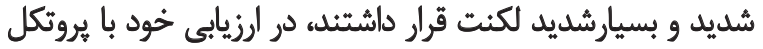

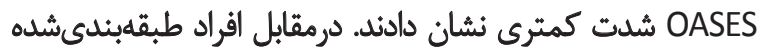

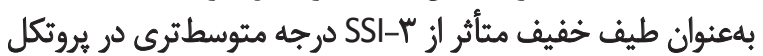
OASES

\section{نتيجليرى}

بلددليل اينكه هدف اين مطالعه مقايسه نمرات كيفيت زندكى إنى إني

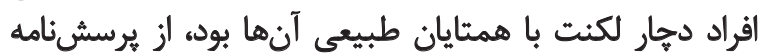

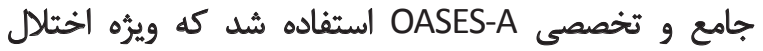
لكنت است. با توجه به نتايج بهدست آمدآمده مشخص شاد شد كيفيت

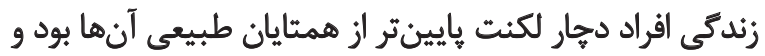

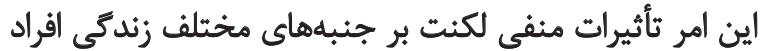

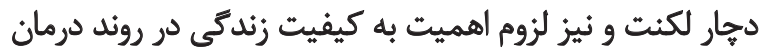

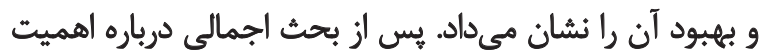

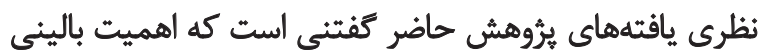

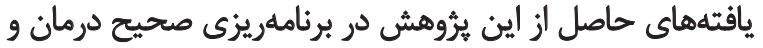

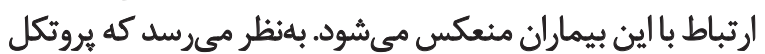

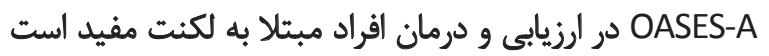

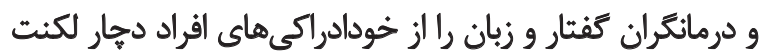

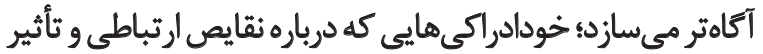
لكنت بر كيفيت زندگى آنهاست.

Loesgow

از محدوديتهاي اين يُروهش تعداد كم آزمودنى هاي مؤنث مبتلا

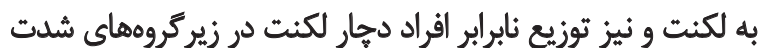
ل كنت و تجربه و نكرش ارتباطى بودي

بيشنينهانها

انجام مطالعات مشابه در فرهنكَهاى مختلف كه ارزشهاى

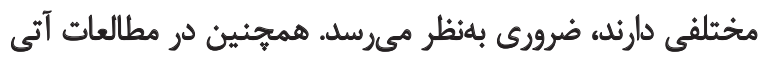




\section{References}

[1] Shafiei B, Salehi A, Karimi H, Yadegari F, Sima Shirazi T, Shafiei M. [The validity and reliability of the Farsi version of "the overall assessment of the speaker's experience of stuttering-adult" (OASES-A) Questionnaire (Persian)]. Research in Rehabilitation Science. 2012; 1(1):49-56.

[2] Bloodstein, O, Ratner NB. A handbook on stuttering. Boston: Thomson Delmar Learning Publication; 2008.

[3] American Speech-Language-Hearing Association. Scope of practice in speech-language pathology [Internet]. 2016 [Cited 18 Apr. 2016]. Available from: http://www.asha.org/policy/SP200700283.htm.

[4] Shafiee B, Mehralian Z. [Disfluency disorders in children's speech (Persian)]. $11^{\text {th }}$ ed. Isfahan: Isfahan University of Medical Sciences; 2003.

[5] Cooper EB. Chronic perserevative stuttering syndrome: A Harmful or helpful construct? American Journal of Speech-Language Pathology 1993; 2(3):11-15. doi: 10.1044/1058-0360.0203.11

[6] Klompos M, Ross E. Life experiences of people who stutter and the perceived impact of stuttering on quality of life: Personal accounts of south African individual. Journal of Fluency Disorder. 2004; 29(4):275-305. doi: 10.1016/j.jfludis.2004.10.001

[7] Bramlet ER, Bothe KA, Franic MP. Using preference-based measures to assess quality of life in stuttering. Journal of Speech, Language \& Hearing Research. 2006; 49(2):381-94. doi: 10.1044/1092-4388(2006/030)

[8] Yaruss JS, Quesal RW. Stuttering and the international classification of functioning, disability, and health (ICF): An update. Journal of Communication Disorders. 2004; 37(1):35-52. doi: 10.1016/s0021-9924(03)00052-2

[9] Franic DM, Bothe AK. Psychometric evaluation of conditionspecific instruments used to assess health-related quality of life, attitudes, and related constructs in stuttering. American Journal of Speech-Language Pathology. 2008; 17(1):60-80. doi: 10.1044/1058-0360(2008/006)

[10] Reilly S, Douglas J, Oates J. Evidence based practice in speech pathology. Philadelphia: Gullan Whur Publication; 2004.

[11] Yaruss JS, Quesal RW. Overall Assessment of the Speaker's Experience of Stuttering (OASES): Documenting multiple outcomes in stuttering treatment. Journal of Fluency Disorders. 2006; 31(2):90-115. doi: 10.1016/j.jfludis.2006.02.002

[12] Tamizi Z, Ranjbar F, Yaghmaei F, Alavi-Majd H, Fallahi-Khoshknab M. [Assessment of relationship between quality of life and coping strategies in schizophrenic patients in refer to psychiatric clinics of educational hospitals of Tehran (Persian)]. Journal of Rehabilitation. 2013; 13(4):124-131.

[13] Yaruss JS. Assessing quality of life in stuttering treatment outcomes research. Journal of Fluency Disordorders. 2010; 35(3):190-202. doi: 10.1016/j.jfludis.2010.05.010

[14] Shafiei M, Shafiei B, Karimi H, Moazeni H, Baghali A, Hoseini Sh, Barzegar E. [Comparing the Quality of Life among Individuals with and without Stuttering (Persian)]. Speech \& Language Pathology. 2014; 1(3):69-75.
[15] Mansuri B, Shahbodaghi MR, Tohidast A, Kamali M. [Comparing the quality of life in adults who stutter with their normal counterparts (Persian)]. Journal of Research in Rehabilitation Sciences. 2013; 9(2):318-27.

[16] Craig A, Blumgart E, Tran Y. The impact of stuttering on the quality of life in adults who stutter. Journal of Fluency Disorder. 2009; 34(2):61-67. doi: 10.1016/j.jfludis.2009.05.002

[17] Bragatto EL, Osborn E, Yaruss JS, Quesal R, Schiefer AM, Chiari BM. Brazilian version of the Overall Assessment of the Speaker's Experience of Stuttering-Adults protocol (OASESA). Journal da Sociedade Brasileira de Fonoaudiologia. 2012; 24(2):145-51. doi: 10.1590/s2179-64912012000200010

[18] Manning W, Beck G. The role of psychological processes in estimates of stuttering severity. Journal of Fluency Disorders. 2013; 38(4):356-67. doi: 10.1016/j.jfludis.2014.05.002

[19] Yadegari F, Salehi A. [Differential diagnosis of primary stuttering and normal nonfluency in children referring to saba clinic (Persian)]. Journal of Rehabilitation. 2004; 4(14):45-48.

[20] Karimi Javan G, Nilipour R, Ashayeri H, Yadegari F, Karimlo M. [The role of explicit and implicit memory in stutterers (Persian)]. Journal of Rehabilitation. 2007; 8(30):69-72.

[21] Basi M, Farazi M, Bakhshi E. Evaluation of effects of gradual increase length and complexity of utterance (GILCU) treatment method on the reduction of dysfluency in school-aged children with stuttering. Iranian Rehabilitation Journal. 2016; 14(1):59-62.

[22] Barikroo A, Rezaee N, Yadegari F. [Investigation of the relationship between hand gestures and speech in adults who stutter (Persian)]. Journal of Rehabilitation. 2008; 4(35):16-21.

[23] Farazi M, Gholami Tehrani L, Khodabakhshiy Kolaee A, Shem shadi H, Rahgozar M. [The effect of a combination of cognitive therapy and speech in stutterers (Persian)]. Journal of Thought \& Behavior in Clinical Psychology. 2014; 9(30):37-46. 
